\title{
Utilización de betabloqueadores en el tratamiento de la insuficiencia cardiaca
}

\author{
C. RODRÍGUEZ MORENO, C. DURÁN PARRONDO*, E. G. DONADO BUDIÑO, M. \\ GARCÍA REBOREDO, F. TATO HERRERO, J. TARRAGÓ BOFARULL, **F. LADO \\ LADO, I. RODRÍGUEZ LÓPEZ** \\ Servicios de Farmacología Clínica. *Centro de Atención Primaria de Lalín. **Medicina \\ Interna. Hospital Clínico Univesitario. Santiago de Compostela
}

USE OF BETABLOCKERS ON TREATMENT OF CARDIAC FAILURE

\begin{abstract}
RESUMEN
El conocimiento de la implicación del sistema adrenérgico en la fisiopatología de la insuficiencia cardiaca (ICC) ha justificado una intensa investigación sobre el papel de los betabloqueadores en el tratamiento de esta enfermedad. Recientemente se han publicado varios ensayos clínicos de gran calidad que demuestran claramente que el tratamiento de la ICC sistólica con betabloqueadores disminuye la mortalidad en un 35\%, la frecuencia de hospitalización y mejora la función ventricular. Sin embargo, existen muchas reticencias para su uso debido a que pueden empeorar el cuadro en algunos pacientes, sobre todo al principio del tratamiento. Esto puede evitarse con un cuidadoso comienzo y un progresivo ajuste de dosis. Todavía quedan cuestiones por responder como la elección del betabloqueador, el efecto del tratamiento en la progresión de la enfermedad y su utilidad en pacientes muy graves o muy ancianos
\end{abstract}

PALABRAS CLAVE: Insuficiencia cadíaca. Betabloqueadores. Tratamiento farmacológico.

\begin{abstract}
The rationale for betablocker use in heart failure, based on neuro hormonal physiology, has been established over the past 20 years. Recent trials have shown the unequivocal benefits of betablockers in patients with chronic systolic heart failure. The benefits include improve survival (35\%) reduced need for hospitalization and improve of left ven tricular function. However, betablockers may also make a patient with heart failure worse, especially when treatment begins and there is reluc tance to use betablockade therapy. Complications can generally be avoi ded by starting with extremely low doses and increasing the dose very slowly. Despite this, further questions remain regarding the use of these agents in cardiac failure, including the role in the progresion of the dise ase, the selection of individual betablocker, and the use in very severe disease or very old patients.
\end{abstract}

KEY WORDS: Cardiac failure. Betablocker. Drug treatment.

Rodríguez Moren C, Durán Parrondo C, Donado Budiño EG, García Reboredo M, Tato Herrero F, Tarragó Bofarull J, Lado Lado F, Rodrí guez López I. Utilización de betabloqueadores en el tratamiento de la insuficiencia cardíaca. An Med Interna (Madrid) 2002; 19: 368 -374.

\section{INTRODUCCIÓN}

La utilización de betabloqueadores (BB) en la insuficiencia cardiaca (ICC) sistólica ha sido defendida desde mediados de los 70 cuando algunos ensayos clínicos encontraron un cierto beneficio en parámetros hemodinámicos y clínicos $(1,2)$. Estos datos y un progresivo mejor conocimiento de la fisiopatología de la ICC y de los fenómenos de remodelación cardiaca, animaron la investigación en este campo. Así, en las siguientes décadas, han ido apareciendo ensayos clínicos controlados (ECC) de pequeño tamaño que han mostrado que la administración de BB en pacientes con ICC sistólica inducía una mejoría de los síntomas, una mejor función ventricular y un aumento de la fracción de eyección (3-9). Entre el año 1980 y el 1997 se realizaron un mínimo de 24 ECC con BB en ICC que incluyeron más de 3.141 pacientes con ICC isquémi- ca y no isquémica y 5 revisiones sistemáticas sobre ellos. Entre todos estos resultados, en 1998 había fuertes evidencias de beneficio pero quedaban serias dudas de que realmente consiguieran disminuir la mortalidad global de los pacientes. De hecho, los primeros ECC de cierto tamaño, el Metoprolol in Dilated Cardiomyopathy (MDC trial), el Australia/New Zealand Heart Failure Study (ANZ Trial) y el Cardiac Insuf ficiency Bisoprolol Study (CIBIS) fueron incapaces de demostrar significación estadística en cuanto a las diferencias en mortalidad, aunque sí una clara tendencia a favor del tratamiento. El problema radicaba, según algunos autores (10), en una falta de poder estadístico de los estudios publicados hasta la fecha. Sin embargo, además de la falta de datos definitivos, los BB han sido siempre un grupo de fármacos vistos con reticencias, incluso en indicaciones con datos tan contundentes de beneficio como la cardiopatía isquémica (11). En muchos

Trabajo aceptado: 6 de noviembre de 2001

Correspondencia: Carlos Rodríguez Moreno, Servicio de Farmacología Clínica del Hospital Clínico Universitario de Santiago. Vidán s/n, 15706 Santiago de Compostela 
clínicos persiste la convicción de que los BB serían contraproducentes en una situación (la ICC) donde un corazón insuficiente necesita la activación del simpático para mantener la contractilidad miocárdica y el gasto, y asegurar la perfusión de órganos vitales.

En los últimos años han aparecido parte de los datos que faltaban. Se han publicado resultados de varios ECC de adecuado tamaño que demuestran consistentemente una mejoría significativa en la mortalidad global de los pacientes con ICC sistólica tratados con BB y ha motivado que, aunque persistan reticencias y como veremos, preguntas sin respuesta, los BB sean aceptados como parte del tratamiento estándar de la ICC sistólica $(12,13)$.

\section{JUSTIFICACIÓN FISIOPATOLÓGICA}

La activación del sistema nervioso simpático es una de las anormalidades más importantes en la fisiopatología de la ICC crónica. Los pacientes con fallo cardiaco tienen niveles anormalmente altos de catecolaminas circulantes y este nivel está en proporción con la severidad; a mayor nivel peor pronóstico (14-16). Esto hizo suponer que la activación simpática formaba parte causal de la progresión de la enfermedad y que su interrupción con betabloqueadores podía ser útil en el tratamiento de la enfermedad.

La disfunción sistólica activa precozmente el sistema simpático como mecanismo de compensación para estimular la contracción ventricular, y mantener el flujo a órganos "nobles" o centrales mediante un aumento del gasto cardiaco y de la tensión arterial. Sin embargo, a largo plazo conlleva una serie de hechos nocivos para corazón, riñón y vasculatura: a) en primer lugar, el aumento de gasto aumenta la demanda de oxígeno del miocardio lo que deviene en isquemia miocárdica y aumento del estrés oxidativo; b) la vasoconstricción arterial y venosa aumenta la pre y postcarga suponiendo un esfuerzo adicional en una bomba que falla; c) los efectos directos de la noradrenalina sobre el miocito: hipertrofia y aumento de la muerte celular programada (apoptosis) por acumulación intracelular de calcio, convergen con el sistema renina-angiotensina-aldosterona (RAA) para producir remodelación cardiaca que se caracteriza por cambios deletéreos en la geometría, masa y volumen, que resultan en una cámara menos contráctil y más dilatada. Como efectos adicionales: d) taquicardia y desensibilización (disminución del número y actividad) de receptores $B_{1}$ lo que disminuye aún mas la fuerza de contracción ventricular; e) trastorno del transporte celular y homeostasis de sodio, potasio y calcio lo que origina un problema de descoordinación de la contracción del miocardio y que a su vez conlleva riesgo de arritmias que pueden contribuir a un aumento de la incidencia de muerte súbita; f) activación directa del sistema RAA.

Además, la subsiguiente isquemia sobre este ventrículo dilatado, reduce aún más la contractilidad lo que aumenta la dilatación. Al final, sucesivos episodios de isquemia, muerte celular y dilatación compensadora, pueden resulta en infarto o fallo ventricular completo (17-19).

El tratamiento habitual de primera línea de la ICC sistólica ignora los efectos debidos a esta activación del sistema adrenérgico. Los diuréticos, mejoran el estado hemodinámico pero de hecho, pueden incluso aumentar los problemas neurohormonales; la digoxina, reduce modestamente el tono adrenérgi- co cardiaco pero no afecta la historia natural de la enfermedad; los IECAs, atajan los efectos del sistema RAA pero no el otro pilar de la activación neurohormonal: el sistema adrenérgico.

El bloqueo de la acción de las catecolaminas en miocardio mejora la función de los miocitos, disminuye la apoptosis, reduce el proceso de remodelación cardiaca y reduce el tamaño de las cámaras cardiacas y además podría frenar en parte la activación del sistema RAA y de la vasoconstricción. Un subestudio del ANZ Trial con ecocardiografía demuestra que carvedilol disminuye significativamente el volumen diastólico y sistólico final del ventrículo izquierdo y aumenta la fracción ventricular de eyección lo que expresa una reversión de la remodelación cardiaca (20). Además, todos los estudios posteriores de gran tamaño coinciden en encontrar un aumento de la fracción de eyección ventricular en el grupo tratado con BB.

Se produce también un aumento del número y de la actividad de los receptores beta responsable de un aumento de la reserva inotrópica y de un aumento en la relación fuerza/frecuencia. El efecto bradicardizante mejoraría la relajación diastólica y disminuiría (junto con lo anterior) las necesidades de oxígeno del miocardio.

Todo esto, disminuiría la progresión de la enfermedad, mejoraría la función ventricular y la capacidad para el ejercicio y disminuiría las arritmias y las complicaciones isquémicas $(21,22)$.

\section{ECC DE MEDIANO TAMAÑO Y REVISIONES SISTEMÁTICAS}

Progresivamente hasta 1998 se desarrolla una intensa investigación sobre este problema pero en la que faltan ECC de gran tamaño. Hasta esa fecha, que marcamos como límite por suponer el comienzo de los ECC de gran tamaño descritos más adelante, pueden encontrarse un mínimo de 24 ECC, 4 de ellos de apreciable tamaño (23-26) y al menos 5 revisiones sistemáticas (10,27-30): Recogemos sucintamente las características y resultados de estos trabajos:

MDC (Metoprolol in Dilated Cardiomyophaty) (23). En este ECC se incluyeron 383 pacientes menores de 75 años con cardiomiopatia dilatada idiopática, ICC con fracción de eyección (FE) menor del 40\%, clase funcional según la clasificación de la New York Heart Assoaciation (NYHA) II-IV, y tratamiento convencional y se les asignó a recibir $75 \mathrm{mg} / 12$ horas de metoprolol o placebo con un seguimiento de 12-18 meses. Como objetivo primario se valoró la mortalidad más la necesidad de transplante. En esta variable se encontró una reducción, no significativa, del 34\% (IC 95\% de -6 a 62\%) en el grupo tratado.

CIBIS (Cardiac Insufficiency Bisoprolol Study) (24). Se incluyeron 641 pacientes menores de 75 años con ICC grados III o IV (5\%), FE menor del $40 \%$ y tratamiento convencional, aleatorizados a recibir bisoprolol o placebo y seguidos durante 1,9 años. Se observó una tendencia no significativa de menor mortalidad en el grupo tratado (reducción de riesgo relativo del $20 \%$, IC $95 \%$ de -15 a 46\%). Sí alcanzó significación estadística la mejoría del grado funcional y los ingresos hospitalarios.

USCHFS (United States Carvedilol Heart Failure Study Group) (25). Se trata de un ECC con 4 subprotocolos en función de la gravedad de los pacientes según su tolerancia al 
ejercicio. En total se incluyeron 1094 pacientes con ICC grados II, III y IV con FE menor del $35 \%$ y tratamiento convencional, que fueron aleatorizados a recibir carvedilol o placebo durante 6-12 meses para evaluar mortalidad y hospitalización de causa cardiovascular. La mortalidad total fue un $65 \%$ menor (IC 95\% de 39-80\%; p<0.0001) por lo que se interrumpió el ensayo. También se encontró $27 \%$ reducción en hospitalización por causas cardiovasculares. Este ensayo resulta decisivo en revisiones sistemáticas y metaanálisis posteriores por su tamaño y su llamativamente alta reducción de la mortalidad. Sin embargo, esto último, puede ser debido a un anormalmente bajo número de éxitus en el grupo control (para lo esperable por la gravedad de los enfermos incluidos). Esto supone una crítica importante sobre la validez de ese resultado tan espectacular y dudas sobre la generalización de estos resultados de mortalidad o su comparación con otros ensayos.

ANZ (Australia/New Zealand Heart Failure Study) (26). En este ECC se incluyeron 415 pacientes con ICC crónica estable ligera, de etiología isquémica en tratamiento convencional y se aleatorizaron a recibir carvedilol o placebo durante 19 meses. Se encontró una reducción del $27 \%$ en la mortalidad $(9,6 \%$ vs $12,6 \%)$ y de un $30 \%$ en la hospitalización pero en ambos casos sin significación estadística. No hubo diferencias en los síntomas, capacidad de ejercicio o empeoramiento de ICC.

Posteriormente se han realizado al menos 5 revisiones sistemáticas y metaanálisis:

Zarembski, en 1995 (27), analiza los datos de 11 ECC que valoraban el efecto de los BB en un total de 623 pacientes con ICC. Encontraron un aumento en la fracción de eyección y mejoría de la clase funcional NYHA.

Heidenreich, en 1997 (28), analiza 17 ECC con un total 3039 pacientes con ICC tratados con BB o placebo más el tra- tamiento convencional. Encuentra menor mortalidad por todas las causas y menor muerte cardiaca no súbita pero no encuentra diferencia en muerte súbita cardiaca en el grupo tratado con BB.

Doughty en 1997 (29) incluye en su revisión 24 ECC con 3141 pacientes, y encuentra una reducción del $31 \%$ en la mortalidad y mejoría de la función ventricular.

Lechat, en 1998 (30), realizó un metaanálisis de 18 ECC con BB en ICC sistólica que agrupan a 3.023 pacientes que mostró una disminución del $32 \%$ de la mortalidad total, del $41 \%$ en muerte súbita y del $37 \%$ en hospitalizaciones y mejoría de la fracción de eyección.

Por último, Avezum (10), con 18 ECC, con 2.896 pacientes encuentra disminución de la mortalidad, hospitalizaciones por fallo cardiaco y transplante, y mejora de fracción de eyección. No encontró efecto sobre la duración y tolerancia al ejercicio.

A pesar de estos resultados, en aquellos momentos no había todavía suficientes evidencias que demostraran un beneficio claro sobre la mortalidad. Estas revisiones sistemáticas y metaanálisis eran vistas con precaución básicamente porque: a) los ECC no tenían como variable principal el análisis de la mortalidad global y, por tanto ésta, representaba un hallazgo posterior del metaanálisis, y b) de todos estos ECC destacan por su tamaño los cuatro descritos: dos de ellos que no alcanzan significación estadística en el descenso de la mortalidad (MDC Trial y ANZ Trial), otro no estaba diseñado para detectar mortalidad total como objetivo primario (CIBIS) y el cuarto (USCHFS), como hemos comentado, levantaba suspicacias por sus resultados y era demasiado influyente en los metaanálisis. Esta situación propició la puesta en marcha de varios ECC de gran tamaño cuya variable principal es el análisis de la mortalidad global que comentamos a continuación y que se resumen en la tabla I.

TABLA I

ENSAYOS CLÍNICOS CON BETA BLOQ UEANTES EN LA INSUFICIENCIA CARDÍACA CRÓ NICA

\begin{tabular}{|c|c|c|c|c|c|c|c|}
\hline Ensayo clínico & $\begin{array}{c}\text { CIBIS } \\
1994(29)\end{array}$ & $\begin{array}{c}\text { MDC } \\
1995(23)\end{array}$ & $\begin{array}{l}\text { USCHFS } \\
1996(25)\end{array}$ & $\begin{array}{c}\text { ANZ } \\
1997(26)\end{array}$ & $\begin{array}{l}\text { CIBIS-II } \\
1999(31)\end{array}$ & $\begin{array}{l}\text { M ERIT-HF } \\
1999(32)\end{array}$ & $\begin{array}{l}\text { CO PERNICUS } \\
2000^{*}\end{array}$ \\
\hline Número pacientes & 641 & 383 & 1094 & 415 & 2647 & 3991 & 2289 \\
\hline $\begin{array}{l}\text { Severidad** } \\
\text { M ortalidad } \\
\text { con placebo }\end{array}$ & $\begin{array}{c}\text { III; } \\
F E<40 \%\end{array}$ & $\begin{array}{c}\|-I\| \\
F E<40 \% \\
(17,3 \%)\end{array}$ & $\begin{array}{c}\text { I-IV } \\
\text { FE<35\% } \\
7,8 \% \\
(11,0 \%)\end{array}$ & $\begin{array}{c}11 \\
12,6 \% \\
(18,6 \%)\end{array}$ & $\begin{array}{c}\text { III/IV } \\
\mathrm{FE}<35 \% \\
228 / 1.320\end{array}$ & $\begin{array}{c}I I / I I I \\
F E<40 \% \\
217 / 2.001\end{array}$ & $\begin{array}{c}\text { III/IV } \\
\mathrm{FE}<25 \% \\
\mathrm{NC} / 1.133\end{array}$ \\
\hline $\begin{array}{l}\text { M ortalidad con } \\
\text { beta bloqueante }\end{array}$ & & & $3,2 \%$ & $9,6 \%$ & $\begin{array}{c}156 / 1.327 \\
(11,8 \%)\end{array}$ & $\begin{array}{c}145 / 1990 \\
(7,2 \%)\end{array}$ & $\begin{array}{l}\mathrm{NC} / 1156 \\
(11,4 \%)\end{array}$ \\
\hline $\begin{array}{l}\text { Reducción del } \\
\text { riesgo relativo: } \\
\text { mortalidad total }\end{array}$ & $\begin{array}{c}20 \% \\
\text { NS }\end{array}$ & $\begin{array}{c}34 \% \\
\text { NS }\end{array}$ & $65 \%$ & $\begin{array}{c}27 \% \\
\text { NS }\end{array}$ & $34 \%$ & $34 \%$ & $35 \%$ \\
\hline IC 95\% & $-15 a 46 \%$ & -6 a $62 \%$ & 39a $80 \%$ & & 19 a $46 \%$ & 21 a $66 \%$ & \\
\hline
\end{tabular}

* No publicado todavía, datos preliminares e incompletos.

**Clase funcional de la New York Heart Association. 
RESULTADOS SOBRE MORTALIDAD DE ENSAYOS CLINICOS RECIENTES Y GRANDES

Los dos primeros ECC de adecuado tamaño que analizan como objetivo primario el efecto sobre la mortalidad global de los BB en la ICC sistólica son el CIBIS-II (31) y el MERITHF (32) y que han sido publicados completamente. Ambos ensayos son de gran poder y rigor metodológico, coinciden en sus resultados y refuerzan los hallazgos de los metaanálisis previos. De hecho, ambos (junto con el COPERNICUS) han sido interrumpidos prematuramente porque el beneficio con el tratamiento con BB era evidente y por tanto no resultaba ético mantener el grupo control. En la actualidad, hay otros dos ensayos terminados que abordan este problema pero que no han sido publicados (COPERNICUS y BEST) y varios que siguen en marcha con diferentes objetivos (véase más adelante):

CIBIS-II (Cardiac Insufficiency Bisoprolol Study-II) (31). En este ensayo clínico se incluyeron 2.647 pacientes con ICC en clase funcional III (la mayoría) y IV y fracción de eyección menor del $35 \%$, que estaban tomando diuréticos de asa, IECAs y un 50\% digoxina con un seguimiento de 1,3 años. Fue interrumpido prematuramente por reducción significativa de la mortalidad total en un $34 \%$ (IC $95 \%$ de 19 a $46 \%$; $\mathrm{p}<0,0001)$. También encuentra reducciones significativas en cuanto a mortalidad cardiovascular, muerte súbita (44\%) y número de hospitalizaciones por fallo cardiaco (20\%).

El mayor efecto se encontró en pacientes en clase funcional III con enfermedad isquémica. De clase IV sólo se habían incluido pacientes estables (como en todos los ensayos). El mayor efecto sobre la mortalidad fue por muerte súbita (42\%) y menos en el de fallo de bomba (descenso de $26 \%$, IC $95 \%$ 0,48-1,14 NS).

MERIT-HF (Metoprolol Randomised Intervention Trial in Heart Failure) (32). Se incluyeron 3991 pacientes con insuficiencia cardiaca en clase II-IV y FE menor del 40\%, estabilizados con tratamiento estándar y aleatorizados a recibir metoprolol en formulación retardada o placebo. El seguimiento medio fue de un año. El ensayo también fue interrumpido por menor mortalidad en el grupo tratado $34 \%$ (IC $95 \%$ entre $21 \%-52 \%)$, menor muerte súbita $(41 \%)$ y menor muerte por fallo cardiaco (49\%) (IC 95\% de 21\%-66\%). En análisis estratificados predefinidos se observó que existía beneficio también en pacientes con IAM previo, HTA o diabetes.

COPERNICUS (Carvedilol Prospective Randomised Cumulative Survival Trial) (33). Este ensayo compara carvedilol y placebo en 2289 pacientes con ICC severa (clase IIIIV) y fracción de eyección menor del $25 \%$ durante 16 meses. El ensayo ha sido interrumpido prematuramente por disminución clara de la mortalidad en el grupo de carvedilol (35\%).

La mortalidad anual en el grupo placebo ha sido del 18,6\% lo que refleja que se trata de pacientes más enfermos que los del MERIT (mortalidad del grupo placebo del 11\%). El riesgo relativo ha sido casi idéntico en los tres ensayos grandes, aunque este refleja, con toda lógica, un mayor efecto absoluto sobre la mortalidad.

BEST (Betablocker Evaluation of Survival Trial) presentado en Atlanta en las sesiones científicas anuales de la Ameri can Heart Association de noviembre de 1999, aleatoriza 2.800 pacientes con ICC clase III-IV en tratamiento estándar a recibir bucindolol o placebo durante 18 meses. Encuentra una reducción del $10 \%$ (no significativa) en la mortalidad por todas las causas. El ensayo no ha sido publicado todavía en su totalidad por lo que no es posible buscar explicaciones a esta aparente discrepancia en los resultados con los ECC previamente descritos.

Posteriormente se han publicado dos metaanálisis que incluían los pacientes del CIBIS-II pero que no incluían ni los del MERIT-HF ni los del COPERNICUS, por haber sido realizados antes de la publicación de estos ensayos. La primera de ellas (34) incluye 25 ECC en pacientes con ICC aleatorizados a BB o placebo que incluyen 6511 pacientes y 810 muertes. El efecto global es que los BB reducen la probabilidad de muerte en $36 \%$ (IC 95\% 25\%-45\%). No hay evidencia de heterogeneidad en los resultados ni evidencia de sesgo de publicación. Los autores destacan que los beneficios de los $\mathrm{BB}$ en estos pacientes son incluso mayores que los de un tratamiento tan establecido como los IECAs. Según los datos del MERIT y el CIBIS comparados con ensayos como el SOLVD y los datos propios de este metaanálisis el número de pacientes necesario tratar para ahorrar una muerte es de 74 para IECAs y 29 para BB (34).

En el último metanálisis encontrado (35) Bonet y cols., con 22 ECC y 5849 pacientes concluyen que los BB disminuyen la mortalidad global, la mortalidad cardiovascular, la mortalidad por fallo de bomba y por muerte súbita en un 34$39 \%$ con respecto al tratamiento estándar y que el descenso de la mortalidad es similar en pacientes con cardiopatía isquémica o sin ella.

\section{CONCLUSIONES}

Los ECC publicados demuestran que los BB (bisoprolol, carvedilol y metoprolol precisamente) pueden disminuir la mortalidad de los pacientes con ICC sistólica entre alrededor de un $35 \%$, disminuir el número de hospitalizaciones, mejorar la fracción de eyección y la función ventricular izquierda y disminuir la necesidad de transplante. Los efectos sobre la tolerancia al ejercicio son menos claros y contradictorios entre diferentes BB. También está demostrado que disminuye la muerte súbita. Este beneficio es añadido al tratamiento convencional que incluye IECAs.

Los pacientes que podrían beneficiarse de un tratamiento con BB, son aquellos con ICC estable (mínimo 2 semanas sin signos de descompensación incluido mayor necesidad de diuréticos) y disfunción sistólica $(\mathrm{FE}<40 \%)$ recibiendo tratamiento estándar y sin asma, bradicardia o hipotensión. El objetivo es reducir la hospitalización y aumentar la supervivencia pero ser realistas en cuanto a los síntomas.

Esto último quiere decir, que el tratamiento de la ICC sistólica con $\mathrm{BB}$ debe verse como una inversión para obtener beneficios a medio y largo plazo a cambio de unos riesgos hemodinámicos a corto plazo:

A los efectos beneficiosos de los BB se considera que contribuyen, el efecto antiarrítmico, antiisquémico, la disminución de la remodelación cardiaca y la resensibilización de receptores beta. Se observa mejoría de la función ventricular, mayor capacidad para el ejercicio (probablemente diferente según el tipo de $\mathrm{BB}$ ), menor muerte súbita y mortalidad por causas cardiovasculares y por todas las causas. Todo esto representa unos beneficios que se observan a medio-largo plazo. Es importante recalcar que no es habitual observar mejoría clínica hasta las 6-12 semanas. 
La utilización de BB conlleva un riesgo de efectos adversos a corto plazo: empeoramiento de la ICC, bradiarrítmias, prolongación de la conducción intraventricular, hipotensión (aunque se crea tolerancia en tratamiento continuado, sobre todo si se emplean bajas y muy progresivas dosis), empeoramiento de la función renal (hay indicios, en nuestra opinión insuficientes, de que quizá con carvedilol no, pues parece mejorar la hemodinámica renal). Estos riesgos a corto plazo pueden ser minimizados con una lenta y cuidadosa escalada de las dosis y una correcta supervisión del estado del enfermo en los primeros meses. De hecho, en los ECC mencionados la mayoría de los enfermos podía alcanzar dosis óptimas de BB.

La mayoría de los efectos indeseables encontrados en los ECC se presentaban en fases precoces y en buena parte disminuían con el tiempo. Aún así, en el metaanálisis de Avenzum se encuentran diferencias estadísticamente significativas a favor de placebo en mareos $(16,2 \%$ vs $7,5 \%$ de placebo), hipotensión $(4,7 \%$ vs $1,7 \%)$ bradicardia $(5,1 \%$ vs $0,6 \%)$ y fatiga (9\% vs $2,6 \%)$. Sin embargo, en el MERIT la tensión arterial descendió más en el grupo placebo que en el grupo tratado lo que los autores atribuyen a una mejoría del gasto cardiaco con BB. Esta discrepancia puede deberse también a un predominio de ensayos con carvedilol en el metaanálisis de Avenzum

Es imperativo una introducción del BB a dosis muy bajas y un aumento lentamente progresivo de las dosis, en función de la tolerancia del enfermo. Para ello, es imprescindible una cuidadosa monitorización del estado clínico del enfermo y continuar hasta que se alcance la dosis máxima tolerada. Para ello se recomienda que los pacientes estén previamente asintomáticos y estabilizados un mínimo de dos semanas. Es pertinente plantearse hasta qué punto es posible este proceder en nuestro actual sistema sanitario.

En la tabla II se recogen las pautas de dosificación empleadas en los ensayos más importantes y que pueden servir de guía para su utilización en la práctica.

\section{CUESTIONES AÚN SIN RESPUESTA}

Se desconoce el efecto en pacientes mayores de 75-80 años y con función sistólica del ventrículo izquierdo (VI) más o menos preservada (en los ensayos había disfunción sistólica) ni en pacientes con grado IV o grado I (asintomáticos), ni en pacientes con infarto, pues no había gran número de estos pacientes en estos estudios.

Algunas de estas respuestas se plantean en ECC actualmente en marcha como el CAPRICORN (Carvedilol Post Infarct Survival Control in Left Ventricular Dysfunction) con 2800 pacientes con reciente infarto y signos de fallo ventricular izquierdo con o sin síntomas o el CARMEN (Carvedilol ACE Inhibitor Remodeling Mild Heart Failure Evaluation) que pretende comparar BB frente a IECAS en fases muy precoces para valorar el efecto sobre la progresión de la ICC. En este último aspecto, hay consensos que ya reconocen la pertinencia de la utilización de BB en fases precoces arguyendo (en base a los datos del MERIT) que como los síntomas no se relacionan bien con la progresión, es aconsejable que se utilicen BB tanto en el tratamiento antihipertensivo (lo que supondría una notable modificación de la práctica habitual) como en la ICC precoz para prevenir la progresión de la enfermedad (13)

Algunos autores consideran que sobre ICC severa hay datos alentadores pues en el CIBIS-I el más beneficiado fue el grupo con clase IV, el COPERNICUS ha demostrado que carvedilol disminuye la mortalidad en pacientes con ICC de grado IV (el ensayo no ha sido publicado todavía por lo que estos son datos preliminares e incompletos) y por otra parte, los beneficios sobre la mortalidad de los BB devendrían en un mayor efecto precisamente en estos pacientes con mortalidad elevada. En cambio, otros autores señalan, con bastante juicio práctico, que la decisión que implica la utilización de BB en la ICC: mejoría a medio plazo a cambio de riesgos a corto plazo, tiene menos sentido en pacientes graves, que además, son los más proclives a presentar problemas con $\mathrm{BB}$ y por otra parte, en ellos es infrecuente conseguir un periodo asintomático como exige la instauración del tratamiento con BB.

$\mathrm{El}$ efecto de los $\mathrm{BB}$ (como ningún tratamiento en particular) tampoco ha sido evaluado en la ICC diastólica aunque es plausible suponer que al existir un anormal llenado del ventrículo sería beneficioso el enlentecimiento de la frecuencia cardiaca que permitíria un mejor llenado por alargamiento de la

TABLA II

DOSIS Y PAUTAS DE AUM ENTO DE BETA BLO QUEANTES EN LOS GRANDES ENSAYOS CLÍNICOS

\begin{tabular}{|c|c|c|c|c|c|c|c|c|c|c|c|}
\hline \multirow[t]{2}{*}{ BB } & \multicolumn{3}{|c|}{ Dosis inicial } & \multicolumn{7}{|c|}{ Escalada semanal de dosis: dosis diaria total (mg) } & \multirow{2}{*}{$\begin{array}{c}\text { Dosis objetivo } \\
\text { (mg) }\end{array}$} \\
\hline & (mg) & 1 & 2 & 3 & 4 & 5 & 6 & 7 & $8-11$ & $12-15$ & \\
\hline $\begin{array}{l}\text { M etoprolol } \\
\text { (M DC trial) (23) }\end{array}$ & 5 & 10 & 15 & 20 & 50 & 75 & 100 & 150 & NA & NA & $100-150$ \\
\hline $\begin{array}{l}\text { M etoprolol } \\
\text { (M ERIT-HF) (32) }\end{array}$ & $12.5-25$ & NA & 50 & NA & 100 & NA & 200 & NA & NA & NA & 200 \\
\hline $\begin{array}{l}\text { Carvedilol } \\
\text { (USCHFS) (25) } \\
\text { Bisoprolol }\end{array}$ & 3.125 & 6,25 & NA & 12,5 & NA & 25 & NA & 50 & NA & NA & 50 \\
\hline (CIBIS-II)31 & 1,25 & 1,25 & 2,5 & 3,75 & 5 & 5 & 5 & 5 & 7,5 & 10 & 10 \\
\hline
\end{tabular}

$\mathrm{NA}=$ No aumento de dosis

(M odificada de CR Gibbs, M K Davies, GYH Lip. M anagement: digoxin and other inotropes, beta blockers and antiarrhytmic and antithrombotic treatment. BMJ 2000; 320:495-497) (39). 
diástole, sobre todo en presencia de fibrilación auricular, por ejemplo estenosis mitral e hipertrofia de VI (hipertensión arterial) y la mejoría en la relación fuerza/frecuencia.

Una cuestión que permanece abierta es, si serían más beneficiosos unos BB frente a otros. La diferencia entre selectivos (metoprolol, bisoprolol) y no selectivos (carvedilol, bucindolol), además de ésta selectividad sobre receptores B cardiacos, está en que los no selectivos tienen efecto vasodilatador y no originan resensibilización de receptores. También se le reconocen al carvedilol propiedades antioxidantes aunque la relevancia de este hecho dista mucho de conocerse (como muchos otros antioxidantes antes de ahora).

Los resultados de los estudios sobre la tolerancia al ejercicio no son concluyentes y a veces contradictorios según el BB utilizado. No han demostrado mejoría en los ensayos con carvedilol y bucindolol pero sí en el MDC y en el MERIT con metoprolol (significativo para duración de ejercicio máximo) y en CIBIS-II con bisoprolol. Se ha sugerido pues, que los agentes selectivos mejorarían la tolerancia al ejercicio y los no selectivos no. Quizá, la diferencia estriba en que los primeros producen un bloqueo más profundo de receptores B1 y B2 y que además no producen una resensibilización de receptores. Sin embargo, tanto en el MDC (metoprolol) como en el USP (carvedilol) hubo mejoría en la calidad de vida medida por los enfermos y por los investigadores.

Por otra parte, se ha sugerido que los agentes que además tienen efecto vasodilatador (carvedilol y bucindolol) tendrían un mayor beneficio que los que no (metoprolol y bisoprolol).
En diferentes estudios, se ha visto que carvedilol tendría más efecto que metoprolol en mejorar la función ventricular izquierda $(33,37)$. El metaanálisis, antes mencionado de Bonet y cols (35) señala también en sus resultados, que la reducción global en la mortalidad es mayor con BB vasodilatadores que con los no vasodilatadores ( $45 \%$ vs $27 \%$; $\mathrm{p}=0,007)$. Sin embargo, éste metaanálisis incluye los grandes ensayos hasta el CIBIS-II, es decir no incluye el MERIT-HF con metoprolol y 3.900 pacientes y en nuestra opinión, los resultados del programa americano con carvedilol (25) pueden haber influido excesivamente en este hallazgo. Precisamente, hemos comentado lo sorprendente de las cifras tan bajas de mortalidad del grupo placebo (y por tanto lo exagerado del beneficio encontrado) en este ECC. En nuestra opinión, la única manera de conocer a ciencia cierta si hay BB con mayor efecto sobre la mortalidad, es mediante la comparación directa entre los dos tipos de BB en un ECC de adecuado tamaño como el COMET (carvedilol vs metoprolol) que compara el efecto sobre mortalidad de ambos BB en pacientes con ICC clase II-III y que está actualmente en marcha. El único ECC aleatorizado comparativo entre carvedilol y metoprolol disponible hasta ahora no encuentra diferencias significativas en cuanto mejoría de la función ventricular izquierda, los síntomas, la capacidad funcional y otras variables referidas al estrés oxidativo (38). En este sentido, es importante resaltar que, en España, todavía no está autorizada la indicación para todos los BB objeto de esta revisión, e incluso en el mercado no están disponibles presentaciones farmacéuticas adecuadas para una correcta escalada de dosis.

\section{Bibliografía}

1. F Waagstein, A Hjalmarson, E Varnauskas, I Wallentin. Effect of chronic beta-adrenergic receptor blockade in congestive cardiomyopathy. $\mathrm{Br}$ Heart J 1975; 37: 1022-36

2. K Swedberg, A Hjalmarson, F Waagstein, I Wallentin. Prolongation of survival in congestive cardiomyopathy by beta-receptor blockade. Lancet $1979 ; 1: 1374-6$

3. EJ Eichhorn, CM Heesch,JH Barnett, et al . Effect of metoprolol on myocardial function and energetics in patients with nonischemic dilated cardiomyopathy: a randomized, double-blind, placebo-controlled study. J Am Coll Cardiol 1994; 24: 1310-20

4. ML Fisher, SS Gottlieb, GD Plotnick, et al. Beneficial effects of metoprolol in heart failure associated with coronary artery disease: a randomized trial. J Am Coll Cardiol 1994; 23: 943-50

5. SL Woodley, EM Gilbert, JL Anderson, et al. Beta-blockade with bucindolol in heart failure caused by ischemic versus idiopathic dilated cardiomyopathy. Circulation 1991; 84: 2426-41

6. MR Bristow, JB O`Conell, EM Gilbert, et al. Dose-response of chronic beta-blocker treatment in heart failure from either idiopathic dilated or ischemic cardiomyopathy. Circulation 1994; 89: 1632-42

7. SL Olsen, EM Gilbert, DG Renlund, DO Taylor, FD Yanowitz, MR Bristow. Carvedilol improves left ventricular function and symtoms in chronic heart failure: a double-blind randomized study. J Am Coll Cardiol 1995; 25: 1225-31

8. M Metra, M Nardi, R Giubbini, LD Cas. Effects of short- and long-term carvedilol administration on rest and exercise hemodynamic variables, exercise capacity and clinical conditions in patients with idiopathic dilated cardiomyopathy. J Am Coll Cardiol 1994; 24: 1678-87

9. H Krum, JD Sackner-Bernstein, RL Goldsmith, et al. Double-blind, placebo-controlled study of the long-term efficacy of carvedilol in patients with severe chronic heart failure. Circulation 1995; 92: 1499-506

10. A Avezum, R Tsuyuki, J Pogue, et al. Beta-blocker therapy for congestive heart failure: a sistematic overwiew and critical appraisal of the published trials. Can J Cardiol 1998; 14; 1045-53.

11. Agustí A, Arnau JM, Laporte JR. Clinical trials versus clinical practice in the secondary prevention of myocardial infarction. Eur J Clin Pharmacol 1994; 46: 95-9

12. I González, JA Gómez, I González. Tratamiento médico de la insuficiencia cardíaca. Información Terapéutica del Sistema Nacional de Salud 2000; 24: 92-105

13. M Packer, J Cohn for the Steering Committee and Membership of the Advisory Council to Improve Outcomes Nationwide in Heart Failure. Consensus Recommendations for the Management of Chronic Heart Failure. Am J Cardiol 1999; 83 (suppl 2A): 1A-38A.

14. WT Abraham, J Hensen, RW Schrier. Elevated plasma noradrenaline concentrations in patients with low-output cardiac failure: dependence on increased noradrenaline secretion rates. Clin Sci 1990; 79: 429-435.

15. M Packer. The neurohumoral hypothesis: a theory to explain the mechanism of disease progression in heart failure. J Am Coll Cardiol 1992; 20: $248-54$.

16. GS Francis, C Benedict, DE Johnstone, et al, for the SOLVD Investigators. Comparison of neuroendocrine activation in patients with left ventricular dysfunction with and without congestive heart failure: a substudy of the Studies of Left Ventricular Dysfunction (SOLVD). Circulation 1990; 82: 1724-9.

17. MR Bristow, RE Hersberger JD Port, et al. Beta-adrenergic pathways in non failing and failing human ventricular myocardium. Circulation 1990; 82 (suppl 1): 12-25

18. WS Colucci. Molecular and cellular mechanisms of myocardial failure. Am J Cardiol 1997; 80: 15L-25L.

19. R Jaffe, MY Flugelman, DA Halon, BS Lewis. Ventricular remodeling: from bedside to molecule. Adv Exp Med Biol 1997; 430: 257-266.

20. RN Doughty, GA Whalley, G Gamble, et al, on behalf of the Australia/New Zealand Heart Failure Research Collaborative Group. Left ventricular remodeling with carvedilol in patients with congestive heart failure due to ischemic heart disease. J Am Coll Cardiol 1997; 29: 1060-6.

21. M Metra, S Nodari, A D`Aloia, L Bontempi, E Boldi, L Dei Cas. A 
rationale for the use of beta-blockers as standard treatment for heart failure. Am Heart J 2000; 139: 511-21 (10689267)

22. WT Abraham. Beta-blockers: The new standard of therapy for mild heart failure. Arch Intern Med 2000; 160: 1237-47 (10809026)

23. F Waagstein, MR Bristow, K Swedberg, et al, for the Metropolol in Dilated Cardiomyopathy (MDC) Trial Study Group. Beneficial effects of metoprolol in idiopathy dilated cardiomyopathy. Lancet 1993; 342: 1441-6.

24. CIBIS investigators and committees. A randomized trial of betablockade in heart failure: The Cardiac Insufficiency Bisoprolol Study (CIBIS). Circulation 1994; 90: 1765-73.

25. M Packer, MR Bristow, JN Cohn, WS Colucci, MB Fowler EM Gilbert NH Shusterman for the US Carvedilol Heart Failure Study Group. The effect of carvedilol on morbidity and mortality in patientes with chronic heart failure. N Engl J Med 1996; 334: 1349-55

26. Australia/ New Zealand Heart Failure Research Collaborative Group. Randomized, placebo-controlled trial of carvedilol in patients with congestive heart failure due to ischaemic heart disease. Lancet 1997; 349 : 375-80

27. DG Zarembski, PE Nolan Jr, Slack MK, et al. Meta-analysis of the use of low doses beta adrenergic blocking therapy in idiopathic dilated cardiomyopathy. Am J Cardiol 1996; 77: 1247-50

28. A Heindenreich, TT Lee, B Massie. Effect of beta-blockade on mortality in patients with heart failure: a meta-analysis of randomized clinical trials. J Am Coll Cardiol 1997; 30: 27-34.

29. RN Doughty, A Rodgers, N Sharpe, S MacMahon. Effects of beta-blocker therapy on mortality in patients with heart failure: a sistematic overwiew of randomized controlled trials. Eur Heart J 1997; 18: 560-565.

30. P Lechat, M Packer S Chalon, M Cucherat, T Arab, J-P Boissel. Clinical effects of beta-adrenergic blockade in chronic heart failure: a metaanalysis of double-blind, placebo-controlled, randomized trials. Circu- lation 1998; 98: 1184-1191

31. CIBIS-II Investigators and Committees. The cardiac insufficiency bisoprolo Study II (CIBIS-II): a randomised trial. Lancet 1999; 353: 9-13

32. MERIT-HF Study Group. Effect of metoprolol CR/XL in chronic heart failure: metoprolol CR/XL randomised intervention trial in congestive heart failure (MERIT-HF). Lancet 1999; 353: 2001-2007.

33. P Fletcher, Jhunter. Beta blockers in heart failure. Aust Prescriber 2000; 23: $120-123$.

34. JGF Cleland, J McGowan, A Clark. The evidence for beta blockers in heart failure. Equals or surpasses that for angiotensin converting enzyme inhibitors. BMJ 1999; 318: 824-25

35. Bonet S, Agustí A, Arnau JM, Vidal X, Diogene E, Galve E, Laporte JR. Beta-adrenergic blocking agents in heart failure: benefits of vasodilating and non-vasodilating agents according to patients characteristics: a meta-analysis of clinical trials. Arch Intern Med 2000; 160: 621-7

36. Metra M, Nodari S, D'Aloia A. Effects of chronic beta-blockade on the haemodynamics and functional capacity of patients with heart failure: a randomized comparison between metoprolol and carvedilol. Eur Heart J 1998; 19 (suppl): 307

37. Di Lenarda A, Sabbadini G, Salvatore L and the Heart-Muscle Disease Study Group. Long-term effects of carvedilol in idiophatic dilated cardiomyopathy with persistent left ventricular dysfunction despite chronic metoprolol. J AM Coll Cardiol 1999; 33: 1926-34.

38. Kukin ML, Kalman J, Charney RH y cols. Prospective, randomized comparison of effect of long-term tratment with metoprolol or carvedilol on symptoms, exercise, ejection fraction, and oxidative stress in heart failure. Circulation 1999; 99: 2645-51

39. Gibbs CR, Davies MK, Lip GYH. Management: digoxin and other inotropes, beta blockers and antiarrhytmic and antithrombotic treatment. BMJ 2000; 320: 495-497. 